Supporting Information for

\title{
Two-Dimensional Lateral Heterostructures Made by Selective Reaction on a Patterned Monolayer $\mathrm{MoS}_{2}$ Matrix
}

Xuewen Wang ${ }^{1}$, Bolun Wang ${ }^{1}$, Yonghuang Wu ${ }^{1}$, Enze Wang ${ }^{1}$, Hao Luo ${ }^{1}$, Yufei Sun ${ }^{1}$, Deyi Fu ${ }^{2, *}$, Yinghui Sun ${ }^{3, *}, K_{a i} \operatorname{Liu}^{1, *}$

1 State Key Laboratory of New Ceramics and Fine Processing, School of Materials Science and Engineering, Tsinghua University, Beijing 100084, China

2 College of Physical Science and Technology, Xiamen University, Xiamen, Fujian 361005, China

3 Beijing Advanced Innovation Center for Materials Genome Engineering, Beijing Key Laboratory for Magneto-Photoelectrical Composite and Interface Science, School of Mathematics and Physics, University of Science and Technology Beijing, Beijing 100083, China

* Corresponding authors. E-mail: dyfu@xmu.edu.cn; yhsun@ustb.edu.cn; liuk@tsinghua.edu.cn 
a

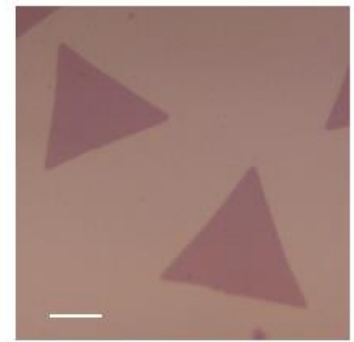

d

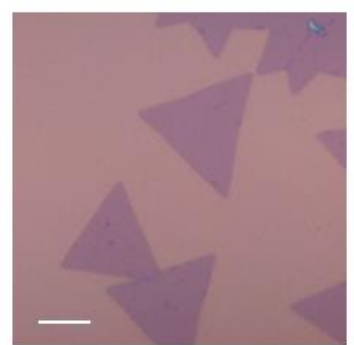

b

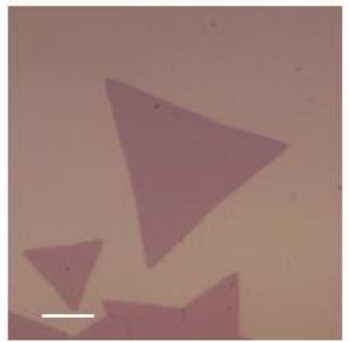

e

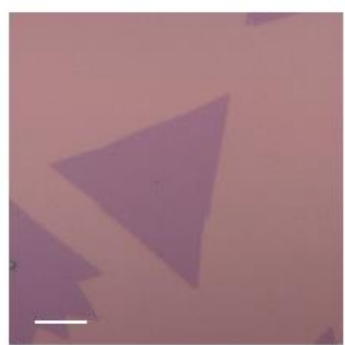

c

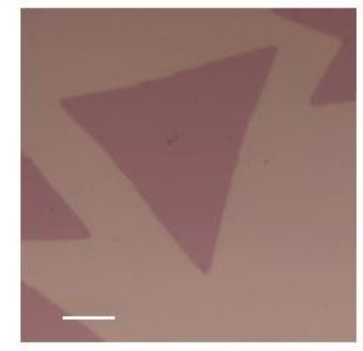

f

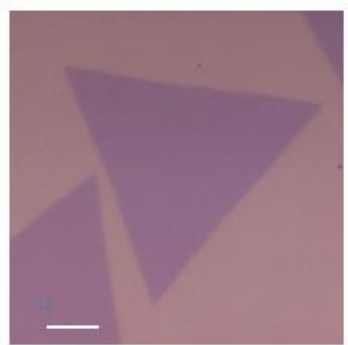

Figure S1. $\mathrm{MoS}_{2}$ synthesis. a-f, Typical optical images of monolayer $\mathrm{MoS}_{2}$ crystals with lateral sizes from 20 to $50 \mu \mathrm{m}(20-30 \mu \mathrm{m}$ a,d, $30-40 \mu \mathrm{m} \mathbf{b}, \mathbf{e}, 40-50 \mu \mathrm{m}$ c,f). Scale bars: $10 \mu \mathrm{m}$. 


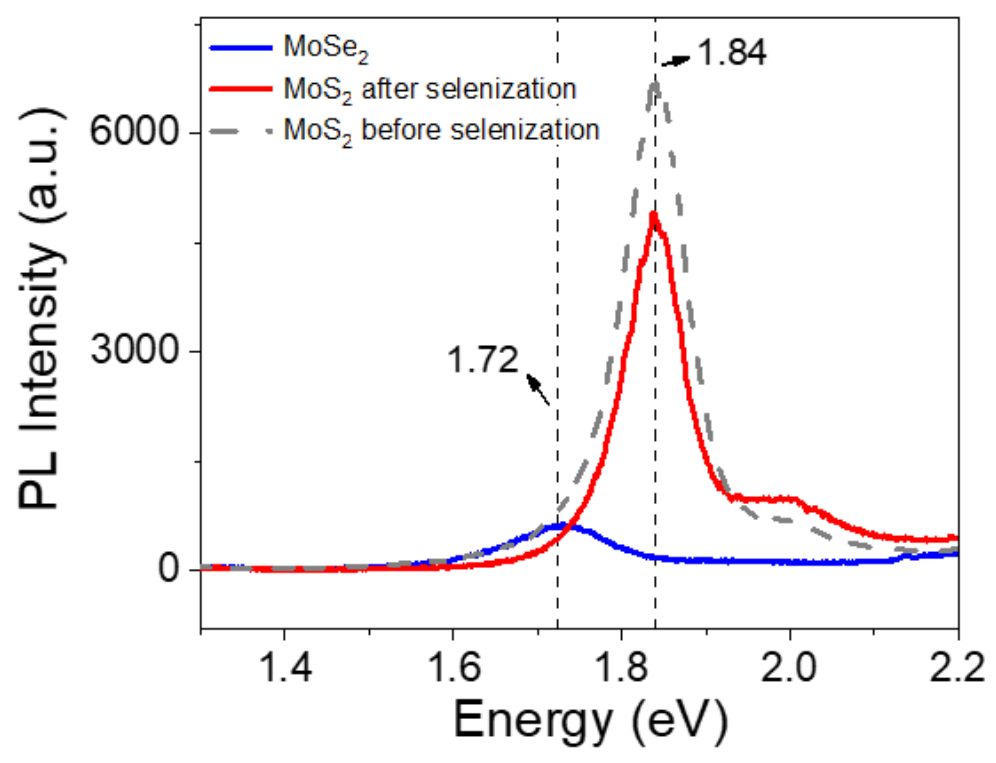

Figure S2. Photoluminescence (PL) spectra of the heterostructure before and after selenization. 
a

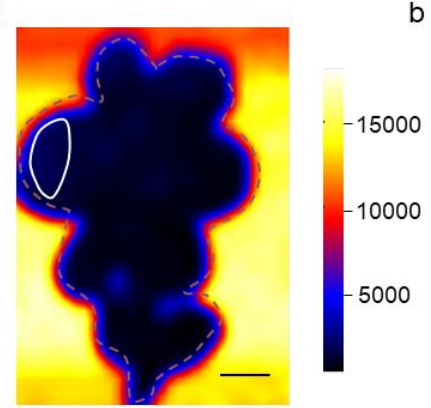

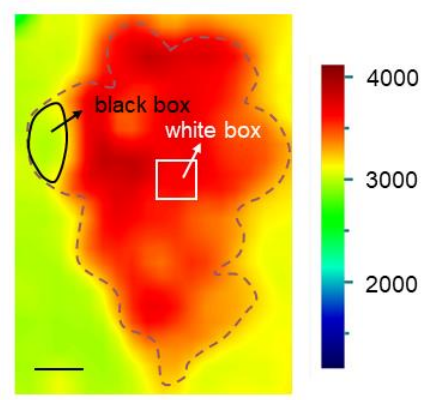

c

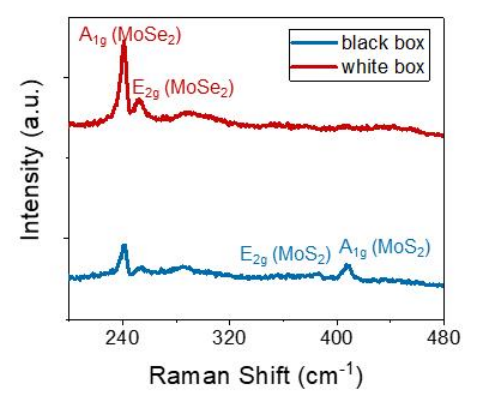

Figure S3. Raman characterization of the heterojunction. a, Raman mapping of the sample after selenization between $386 \mathrm{~cm}^{-1}$ and $405 \mathrm{~cm}^{-1}\left(\mathrm{E}_{2 \mathrm{~g}}\left(\mathrm{MoS}_{2}\right)+\mathrm{A}_{1 \mathrm{~g}}\left(\mathrm{MoS}_{2}\right)\right)$. b. Raman mapping of the sample after selenization at $289 \mathrm{~cm}^{-1}\left(\mathrm{E}_{2 \mathrm{~g}}\left(\mathrm{MoSe}_{2}\right) . \mathbf{c}\right.$, Characteristic Raman spectra of $\mathrm{MoSe}_{2}$ in the black and white boxes in (b). Scale bars, $5 \mu \mathrm{m}$. 

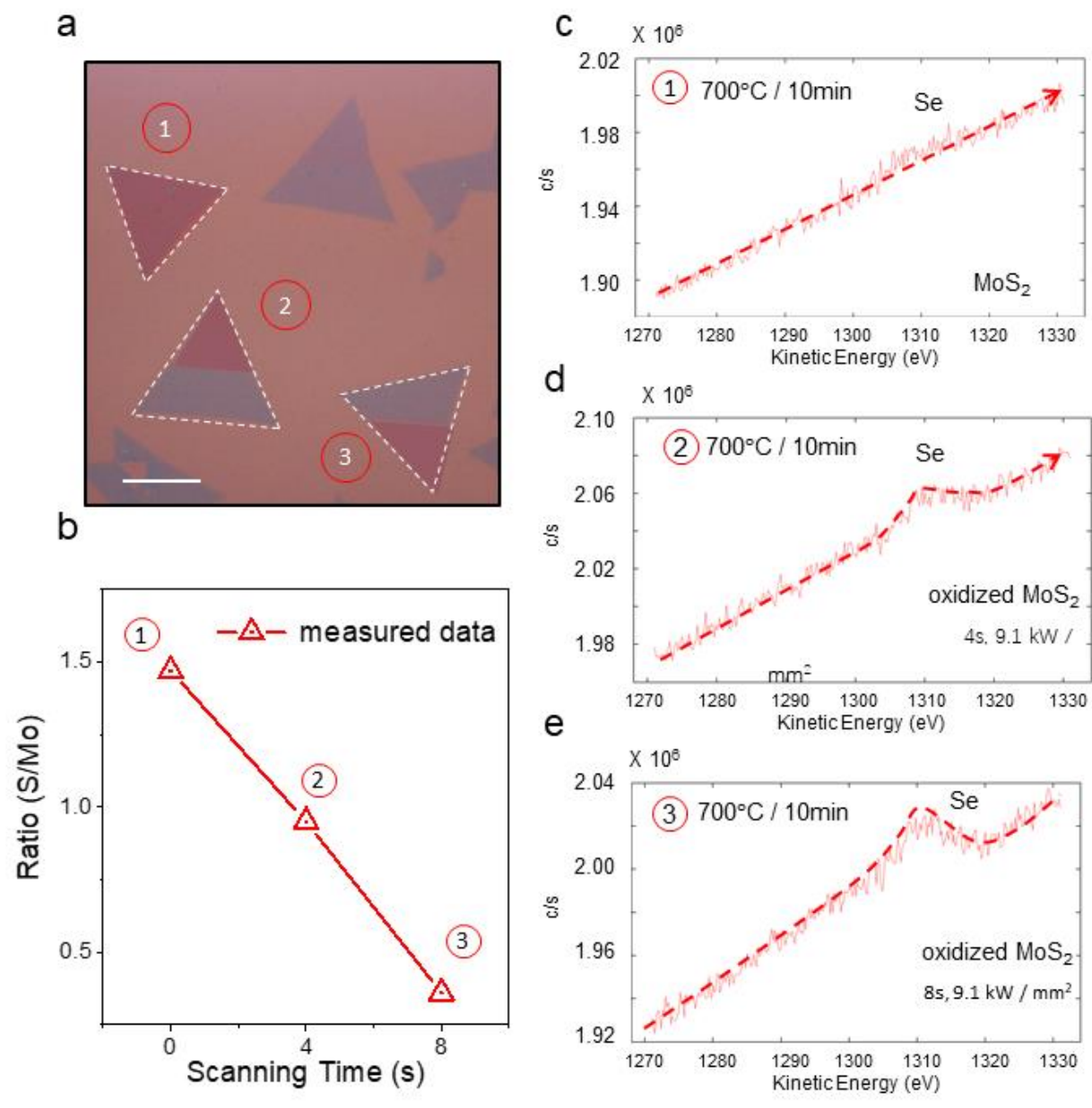

Figure S4. Auger characterization of $\mathrm{LS}^{-\mathrm{MoS}_{2}}$ with different oxidation degrees after selenization. a, Optical image of $\mathrm{MoS}_{2}$ with different oxidation degrees. The image has been colorcoded so that laser-scanned regions appear red. Region 1 (red) represents the sample was scanned at $0 \mathrm{~kW} / \mathrm{mm}^{2}$, while region 2 (red) and 3 (red) represent the samples were scanned at $9.1 \mathrm{~kW} / \mathrm{mm}^{2}$ for $4 \mathrm{~s}$ and $8 \mathrm{~s}$ respectively. Laser wavelength: $532 \mathrm{~nm}$. Growth parameters: $T_{\mathrm{s}}: 700{ }^{\circ} \mathrm{C}, T_{\mathrm{Se}}: 340{ }^{\circ} \mathrm{C}$, $10 \% \mathrm{H}_{2} / 90 \%$ Ar. Scale bar: $10 \mu \mathrm{m} . \mathbf{b}, \mathrm{S} /$ Mo ratio of the three regions in $\mathbf{a}$. The ratio is calculated by relative Auger elemental sensitivity factors. c-d, Corresponding Auger electron spectra for $\mathrm{Se}\left(E_{\mathrm{K}^{-}}\right.$ $1311 \mathrm{eV}$ ) in region $1 \mathbf{c}, 2 \mathbf{d}, 3 \mathbf{e .}$ 

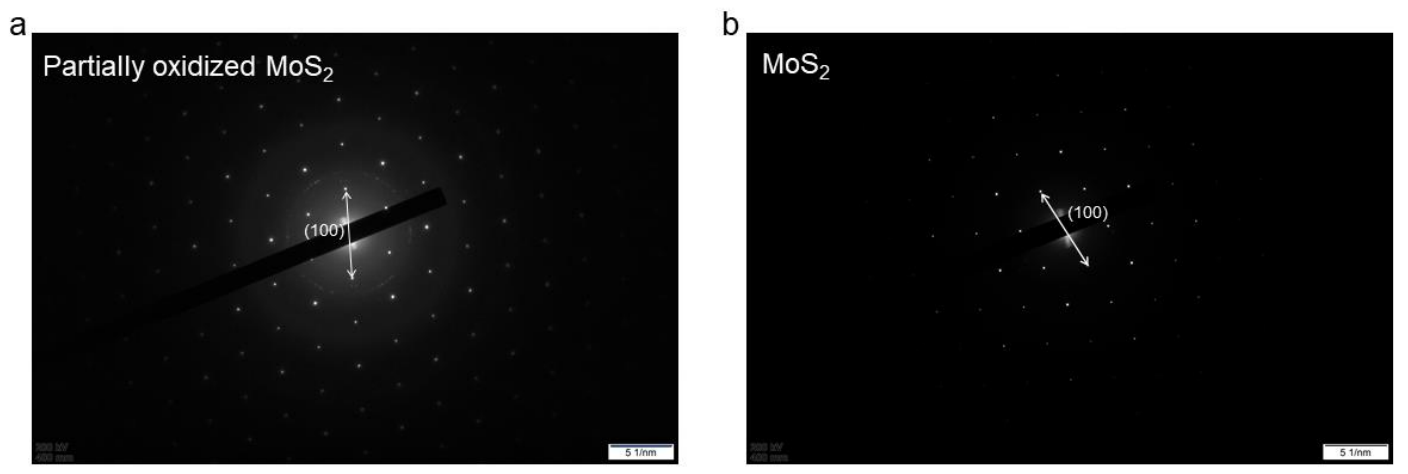

Figure S5. a,b, Diffraction images of partially oxidized $\mathrm{MoS}_{2}$ and pristine $\mathrm{MoS}_{2}$, respectively. The lattice spacing of the polycrystalline ring calculated from (a) is $0.37 \mathrm{~nm}$, close to that of $\mathrm{MoO}_{3}{ }^{1}$, confirming that the laser does oxidize the $\mathrm{MoS}_{2}$ surface into polycrystal $\mathrm{MoO}_{3}$. The hexagonal lattice structure with the lattice spacing of $0.27 \mathrm{~nm}$ in (a) and (b) agrees with the (100) plane of $\mathrm{MoS}_{2}{ }^{2}$. 

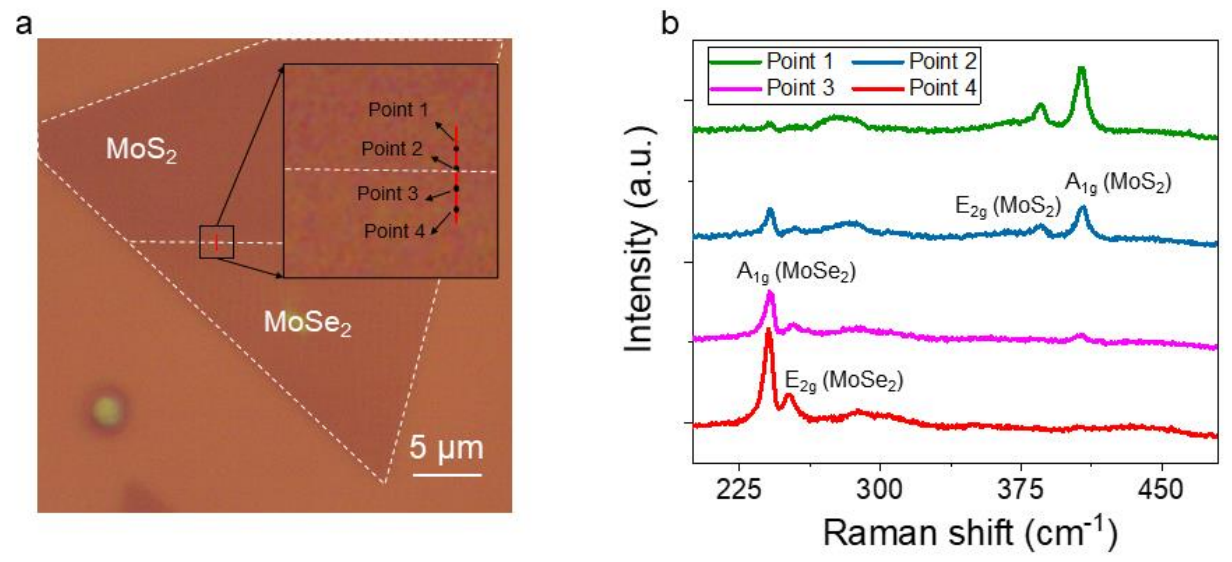

Figure S6. a,b, Optical and Raman characterizations of the transition region between $\mathrm{MoS}_{2}$ and $\mathrm{MoSe}_{2}$ regions, respectively. The four points in (a) are evenly distributed on a straight line with the length of $\sim 0.6 \mu \mathrm{m}$ near the $\mathrm{MoS}_{2} / \mathrm{MoSe}_{2}$ boundary, with a distance of $0.2 \mu \mathrm{m}$ between each point. Point 1 indicates a $\mathrm{MoS}_{2}$-dominated product with a tiny amount of $\mathrm{MoSe}_{2}$, point 2 a mixing product with both $\mathrm{MoS}_{2}$ and $\mathrm{MoSe}_{2}$, point 3 a $\mathrm{MoSe}_{2}$-dominated product with a tiny amount of $\mathrm{MoS}_{2}$, and point 4 a pure $\mathrm{MoSe}_{2}$ product. 
a

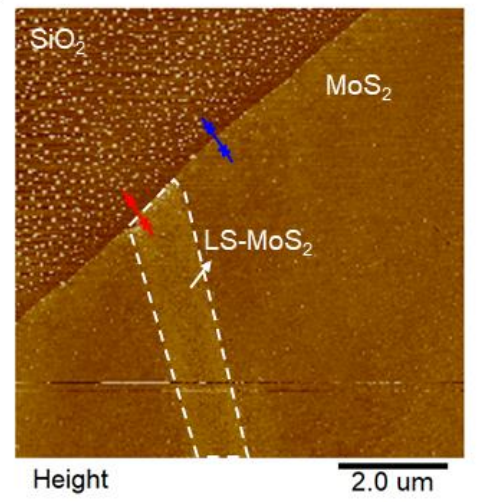

b

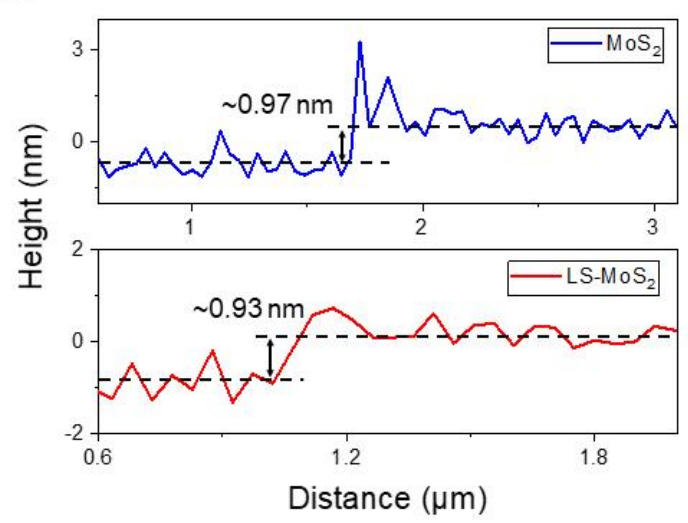

Figure S7. AFM characterization. a,b, AFM topology image and thickness measurements of $\mathrm{MoS}_{2}$ and LS-MoS 2 regions. 


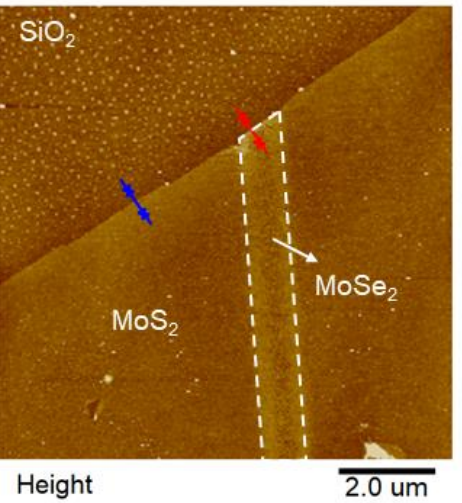

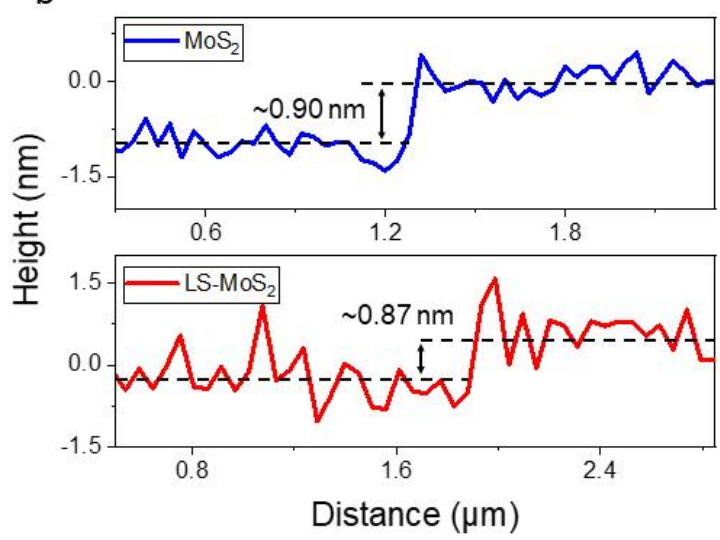

Figure S8. AFM characterization. a,b, AFM image and thickness measurements of $\mathrm{MoS}_{2}$ and LS$\mathrm{MoS}_{2}$ regions after selenization. 

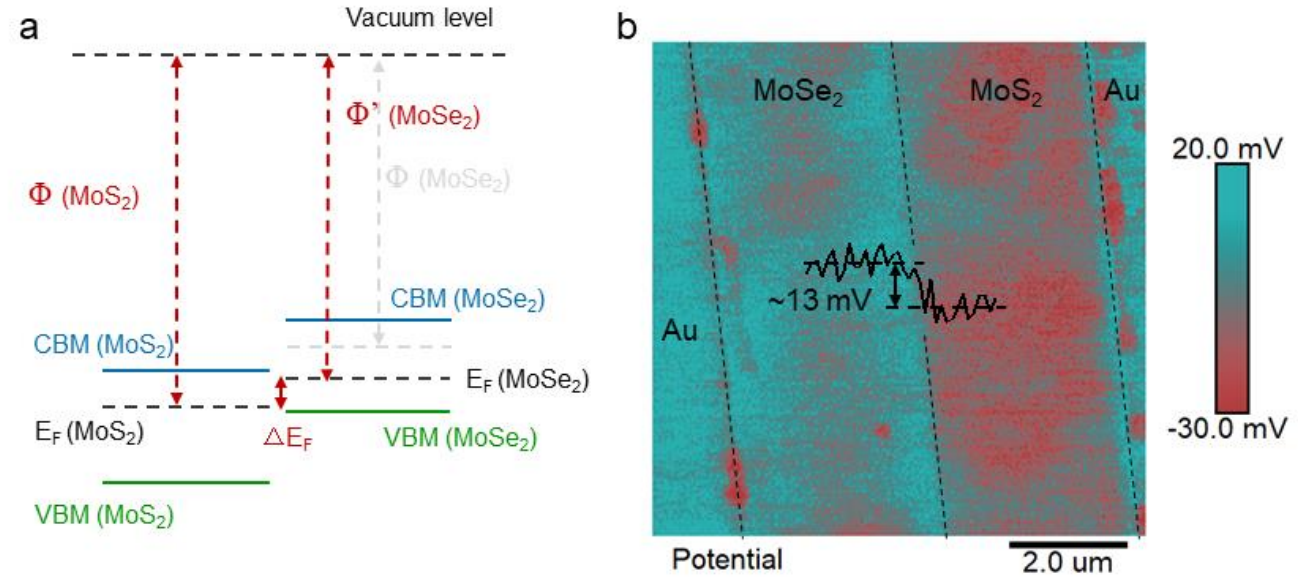

Figure S9. a, Schematic band diagram of $\mathrm{MoS}_{2}$ and $\mathrm{MoSe}_{2}$. b, KPFM image of a MoSe $2 / \mathrm{MoS}_{2}$ heterostructure. 
a

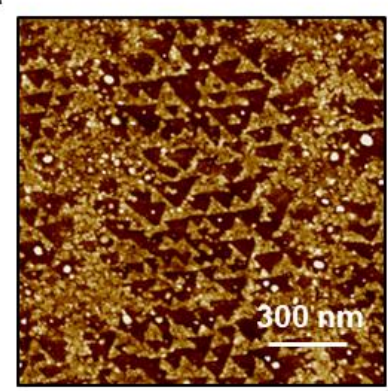

b

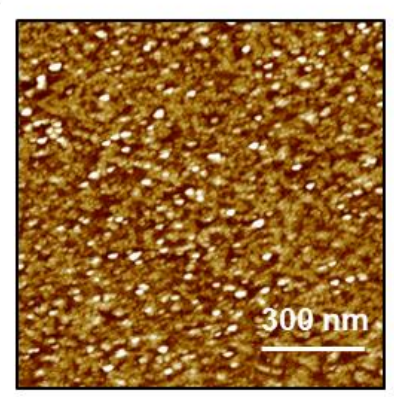

C

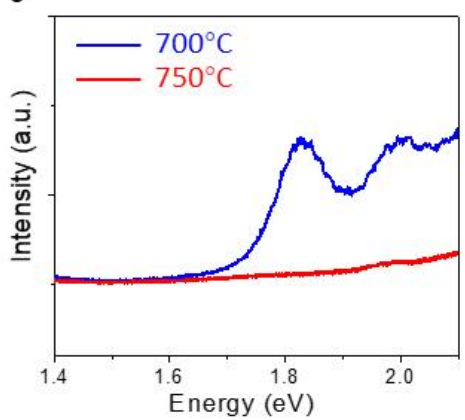

Figure S10. Characterization of selective selenization. a, AFM images of laser-scanned regions under pure $\mathrm{Ar}$ a, and $10 \% \mathrm{H}_{2} / 90 \% \mathrm{Ar} \mathbf{b}$, atmospheres. c, Photoluminescence (PL) spectra of laserscanned regions after selenization at $700{ }^{\circ} \mathrm{C}$ (blue line) and $750{ }^{\circ} \mathrm{C}$ (red line). 


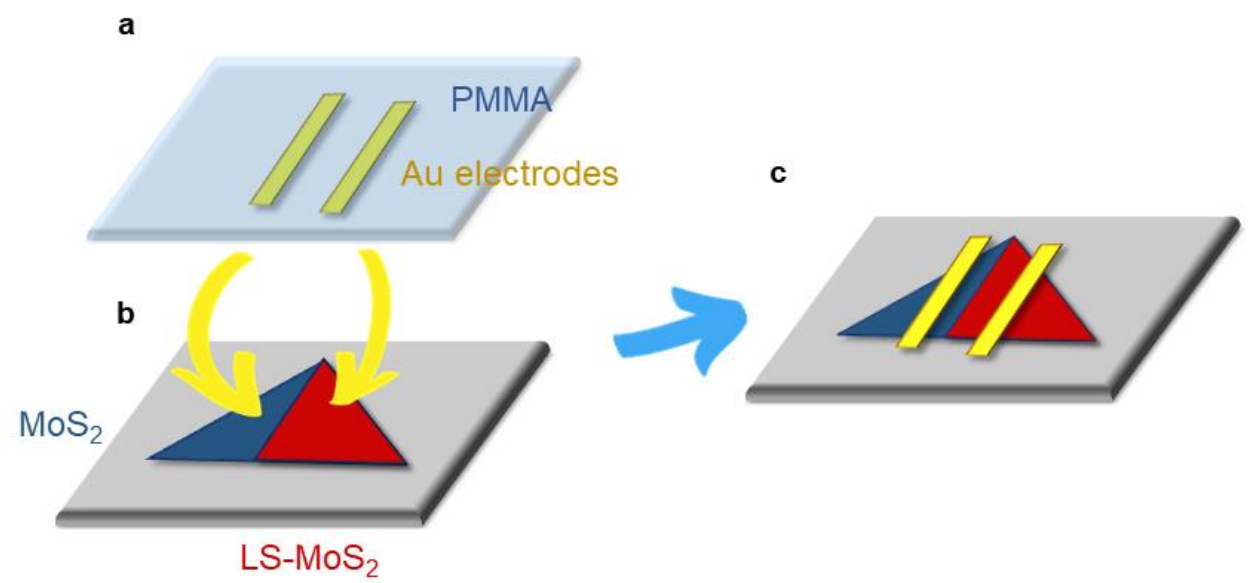

Figure S11. Schematic illustration of device fabrication. a, Transferring Au electrodes via PMMA. b, Alignment. c, Removing the PMMA. 


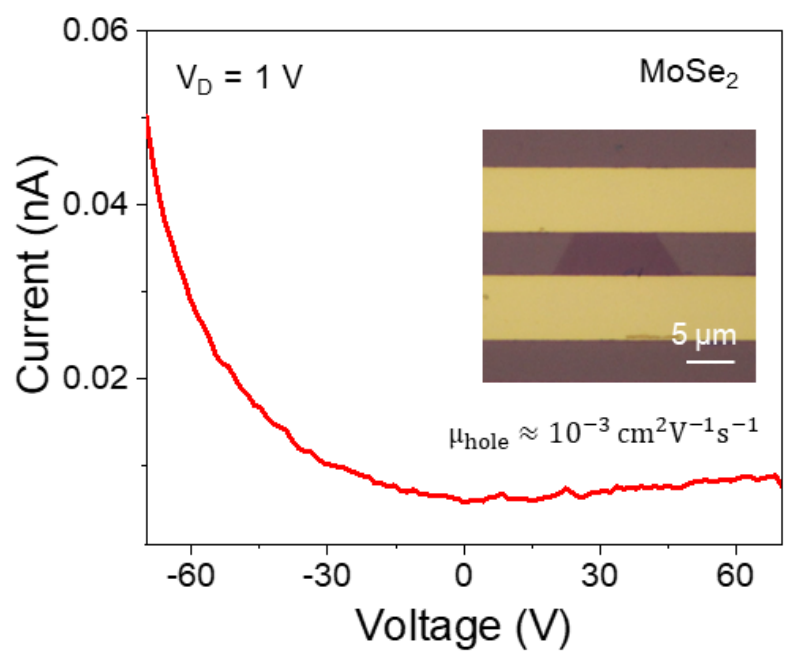

Figure S12. $\mathrm{I}_{\mathrm{D}}-\mathrm{V}_{\mathrm{G}}$ curve of the $\mathrm{MoSe}_{2}$ device at $\mathrm{V}_{\mathrm{D}}=1 \mathrm{~V}$. Inset: optical image of the tested device. 


\section{REFERENCES}

(1) Xia, T. A.; Li, Q.; Liu, X. D.; Meng, J. A.; Cao, X. Q. Morphology-Controllable Synthesis and Characterization of Single-Crystal Molybdenum Trioxide. J. Phys. Chem. $B$ 2006, 110, 2006-2012.

(2) Lee, Y. H.; Zhang, X. Q.; Zhang, W. J.; Chang, M. T.; Lin, C. T.; Chang, K. D.; Yu, Y. C.; Wang, J. T. W.; Chang, C. S.; Li, L. J.; Lin, T. W. Synthesis of Large-Area $\mathrm{MoS}_{2}$ Atomic Layers with Chemical Vapor Deposition. Adv. Mater. 2012, 24, 2320-2325. 